\title{
EFFECT OF MICRO PROTRUSIONS ON THE PERFORMANCE OF ELLIPTICAL JOURNAL BEARINGS OPERATING WITH COUPLE STRESS LUBRICANT
}

\author{
Basim Ajeel ABASS ${ }^{1}$, Mushrek A. MAHDI ${ }^{2}$ \\ ${ }^{1}$ University of Babylon, College of Engineering, Mechanical Engineering Department, Babylon, Iraq \\ Eng.basim.ajeel@uobabylon.edu.iq \\ ${ }^{2}$ University of Babylon, College of Engineering/Al-Musayab, Automobile Engineering Department, Babylon, \\ Almusyab-Iraq. msb.mushrek.alawi@uobabylon.edu.iq
}

\begin{abstract}
Elliptical bearing is characterized by low induced temperature and high stability at higher speed in comparison with the cylindrical journal bearing. The performance of such bearings can be affected by the type of oil used and its surface properties. The main goal of this work is to study the surface micro protrusion effect on the steady state operation of elliptical journal bearing lubricated with couple stress lubricant. The modified Reynolds' equation to take into account the effects of couple stress lubricant and micro protrusions of the bearing surfaces is solved using finite difference method. The governing equations were solved using a prepared FORTRAN computer program. Pressure distribution, load carrying capacity and coefficient of friction have been studied for a bearing in laminar flow regime. The mathematical model was validated by comparing pressure results for the elliptical bearing of the present work with that obtained by Hussain et al.(1996). The obtained results demonstrate that the lubricant pressure and the load carried by the bearing decrease for the bearing with higher ellipticity factor while the load carried by the bearing increases for the bearing with higher protrusions or when using a couple stress fluid with higher couple stress variable.
\end{abstract}

Keywords: elliptical journal bearing, hydrodynamic lubrication, micro-protrusions, couple stress lubricant, non-Newtonian fluids

\section{INTRODUCTION}

Journal bearings are extensively used in industrial application due to its effectiveness and manufacturing simplicity. These types of bearing suffer from instability at higher speeds which lead to the advent of using bearings with noncircular shapes and different types of lubricants. Elliptical bearing is one of those bearings suggested to treat such problem in addition to its lower oil film temperature in comparison with the cylindrical bearings. It was commonly used in turbo-sets of small and medium ratings, steam turbines, and generators. The performance of cylindrical journal bearing lubricated with couple stress fluid under steady state condition was studied by Wang et al. [1], and Maet et al. [2]. It was found that using couple stress fluid in lubricating the journal bearing improves the performance of such bearings. Chiang et al. [3] considered the effects of couple stress and surface roughness on the journal bearing performance. It was observed that the surface roughness effect is dominant in long bearing approximation while it in reverse trend in case of transverse or longitudinal roughness of the journal bearing. Crosby et al. [4] studied the static and dynamic characteristics of two-lobe journal bearings lubricated with couple-stress fluids. The results obtained show an enhancement in stability of the bearing when it was lubricated with couple stress lubricant. In another work, Rahmani et al.[5] explored the effect of lubricating elliptical bore journal bearing with granular particles on static and dynamic performance of such bearing. It was found that the larger size particles enhance the bearing performance better than the smaller one. It was also concluded that the load carrying capacity the side leakage and the coefficient of friction are negatively affected by the ellipticity factor of the bearing. Chetti [6] investigates the effect of couple stress fluid on the elastohydrodynamic performance of an offset journal bearing. It was observed that the bearing characteristics were greatly affected by the elastic deformation of the bearing surfaces. The effect of oil film temperature on the performance of elliptical journal bearing considering different loads and speeds was studied numerically by Chauhan et al. [7] and experimentally by Singla and Chauhan [8] and [9]. The obtained results depict that the temperature and pressure at the mid plane of the bearing increased for higher journal speed and bearing eccentricity ratio while the oil film temperature decreases for the bearing with higher ellipticity factor. A comparative study of thermal behaviour of elliptic, offset halves, and orthogonally displaced non circular journal bearings were performed by Chauhan et al. [10] and Singla and Chauhan [11]. It was observed that the 
cavitation area reduced along the circumference resulting in lower thermal degradation of an oil in noncircular journal bearing as compared to circular one. Dang et al.[12] studied the effect of using nano lubricant with different nano particles and different oil grades on the steady state thermohydrodynamic performance of elliptical journal bearing. It was found that adding $2 \%$ wt of $\mathrm{TiO}_{2}$ and $\mathrm{CuO}$ to the (AW68) base oil enhances the load carrying capacity of the bearing when it was working at an eccentricity ration of 0.7 . The effect of surface roughness on the performance of elliptical journal bearing was studied by Mishra [13] and [14]. Load carrying capacity, friction force, flow into the bearing and the side leakage were the most important bearing parameters analysed. It was recognized that the isotropic roughness of the bearing surfaces has no effect on flow rate into the bearing and the side leakage flow while it increases the load carrying capacity and the friction force. EL-Said et al. [15] studied the effect of surface micro protrusion on the steady state and dynamic performance of noncircular journal bearing. It was found that the three-lobe bearing with protruded bushing is superior in comparison with that of smooth surface bearing from the steady state performance point of view. Faria [16] analysed steady state and dynamic performance of an elliptical journal bearing using finite element method with four nodes isoperimetric rectangular finite elements. The effect of the bearing ellipticity on the load carrying capacity and other performance parameters has been demonstrated and the obtained results bringing some technical data about the behaviour of such bearings. Chetti [17] analysed the steady state laminar and turbulent performance of two-lobe journal bearings lubricated with couple stress fluid. Numerical findings show that the couple stress lubricant strongly affects the performance of such bearing in both regimes. Ukani and Mathur[18] studied the static and dynamic characteristics of elliptical journal bearing lubricated with couple stress lubricant. The results obtained for such bearing were compared with that lubricated with Newtonian lubricant. The obtained results show that the load carrying capacity increases while the attitude angle decreases when the bearing lubricated with couple stress fluid with higher couple stress parameter. Awati and Kengangutti [19] (performed thermo-hydrodynamic analysis for rough journal bearing lubricated with couple stress fluid. It was noted that using lubricants with couple stress for such bearing increases the load carrying capacity and decreases friction coefficient and oil film temperature. Rohilla et al. [20] showed that the effect of couple stress fluid and the elastic deformation of the bearing surface have a significant effect on its performance. It was also shown that the bearing stability increases as the elastic coefficient increases. Dass et al. [21] examined the associated effects of viscosity, velocity slip and the couple stress fluid on the performance of journal bearing. The obtained results indicated that including the effect of slip improves the load carrying capacity and decreases the friction coefficient significantly.

Kumar et al. [22] studied thermohydrodynamic performance of elliptical journal bearing use in steam turbine numerically and experimentally. Finite element method was used to solve the governing equations. A good agreement between the numerical and experimental analysis has been obtained. Bhaskera et al. [23] studied thermal and isothermal performance of elliptical journal bearing lubricated with hydrol 68 lubricating oil at constant journal speed and variable loads. The temperature and pressure have been evaluated along the mid plane. The present work is an attempt to study the performance of elliptical journal bearing under combined effects of surface protrusions and couple stress fluid which is not studied previously.

\section{MATHEMATICAL MODEL}

The problem considered in the present work is statically loaded finite length elliptical journal bearing with the physical geometry shown in figure 1. The geometrical and operating parameters of such bearing can be shown in table (1). The bearing is mathematically modeled in order to study the effect of micro protrusions on its performance when it is lubricated with couple stress lubricant.

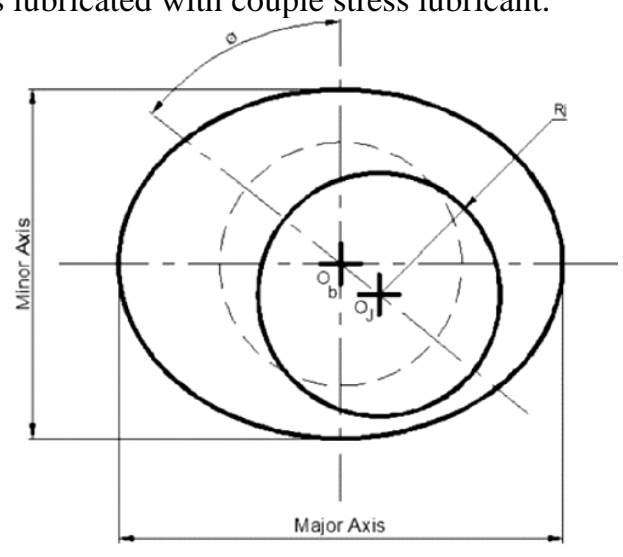

Fig. 1. Elliptical journal bearing

\subsection{Governing Equations and Boundary conditions}

The main governing equations used to analyze the problem of the present work can be summarized as follows:

\section{Reynolds Equation}

Lubricant containing long chain polymers cannot be treated as Newtonian fluid which can be treated as a couple stress fluid characterized by couple stress parameter $\bar{l}$. The couple stress parameter is equal to zero for Newtonian fluid while it may takes a wide range of values for couple stress fluid. The generalized Reynolds' equation 
used to determine the pressure distribution through the bearing gap for laminar flow can be expressed as [17]

$\frac{\partial}{\partial x}\left(\frac{I(h, l)}{\mu} \frac{\partial P}{\partial x}\right)+\frac{\partial}{\partial z}\left(\frac{I(h, l)}{\mu} \frac{\partial P}{\partial z}\right)=6 U \frac{\partial h}{\partial x}$

Where:

$$
I(h, l)=h^{3}-12 h l^{2}+24 l^{3} \tanh \left(\frac{h}{2 l}\right)
$$

Table1. Bearing geometrical and operating conditions [9]

\begin{tabular}{|l|l|}
\hline Bearing Length & $\mathrm{L}=100 \mathrm{~mm}$ \\
\hline journal Diameter & $\mathrm{D}=100 \mathrm{~mm}$ \\
\hline Bearing major axis & $100.4 \mathrm{~mm}$ \\
\hline Bearing minor axis & $100.2 \mathrm{~mm}$ \\
\hline Bearing clearance & $100.4 \mu \mathrm{m}$ \\
\hline Ellipticity ratio & 1 and 1.5 \\
\hline Rotational speed & $\mathrm{N}=3000 \mathrm{rpm}$ \\
\hline $\mathrm{L}$ & $\begin{array}{l}\text { characteristic additives } \\
\text { length, }(\mathrm{m})(\mathrm{l}=\mathrm{n} / \mu)^{0.5}\end{array}$ \\
\hline$\eta$ & $\begin{array}{l}\text { Material constant for the } \\
\text { CSF property }\end{array}$ \\
\hline Atmospheric pressure & $p_{a}=0.0$ \\
\hline lubricant viscosity & $\mu_{o}=0.0277$ pa. $\mathrm{s}$ \\
\hline protrusion height $\left(\mathrm{h}_{\mathrm{p}}\right)$ & $\begin{array}{l}\mathrm{h}_{\mathrm{p}}=0.0(\text { Smooth bearing }), \\
\mathrm{h}_{\mathrm{p}}=\mathrm{H}_{\mathrm{a}} / \mathrm{c}=0.3\end{array}$ \\
\hline $\mathrm{X}$ and $\mathrm{z}$ protrusion widths & $n_{x}=n_{z}=6$ \\
\hline Geometry factor $(\mathrm{m})$ & $\mathrm{m}^{\mathrm{m}}=1$ \\
\hline$\Delta^{*}$ & $\begin{array}{l}\text { Dimensionless variation in } \\
\text { the film thickness }\end{array}$ \\
\hline
\end{tabular}

The dimensionless form of equation (1) can be obtained by using the following nondimensional groups.

$x=R \theta, z=\bar{z} L, l=\bar{l} c, \mu=\mu_{o} \bar{\mu}, h=\bar{h} c$

Then the Reynolds equation (1) can be written in nondimensional form as:

$$
\frac{\partial}{\partial \theta}\left(\bar{I}(\bar{h}, \bar{l}) \frac{\partial \bar{P}}{\partial \theta}\right)+\left(\frac{R}{L}\right)^{2} \frac{\partial}{\partial \bar{z}}\left(\bar{I}(\bar{h}, \bar{l}) \frac{\partial \bar{P}}{\partial \bar{z}}\right)=6 \bar{\mu} \frac{\partial \bar{h}}{\partial \theta}
$$

where:

$$
\bar{I}(\bar{h}, \bar{l})=\bar{h}^{3}-12 \bar{l}^{2} \bar{h}+24 \bar{l}^{3} \tanh \left(\frac{\bar{h}}{2 \bar{l}}\right) .
$$

\section{Oil Film thickness}

The oil film thickness for smooth elliptical journal bearing can be expressed as [12]

$h=C_{v}\left(1+\varepsilon \cos \theta+E_{p} \sin ^{2}(\theta+\phi)\right)$ (5)

where:

$$
E_{p}=\frac{C_{h}-C_{v}}{C_{v}}
$$

$C_{v}, C_{h}$ : vertical and horizontal clearances.

The oil film thickness in dimensionless form can be written as:

$$
\bar{h}=h / C_{v}=1+\varepsilon \cos \theta+E_{p} \sin ^{2}(\theta+\phi)
$$

For elliptical bearing with micro protruded surfaces, the oil film thickness can be expressed as [15]:

$$
\bar{h}=1+\varepsilon \cos \theta+E_{p} \sin ^{2}(\theta+\phi)-\Delta^{*}(\theta, \bar{z})
$$

where:

$\Delta^{*}$ is the oil film thickness variation measured from the stationary bearing surface (dimensionless). Protrusion profile may be modeled mathematically using the following relation [15]:

$$
\Delta^{*}=\left(\frac{H_{a}}{c}\right) \sin ^{2 m}\left[\pi\left(\frac{\theta \cdot n_{x}}{2 \pi}-1\right)\right] \sin ^{2 m}\left[\pi\left(\bar{z} \cdot n_{z}-1\right)\right]
$$

where:

$\mathrm{m}$ is the geometry factor.

$n_{x}, n_{x}$ and $H_{a}$ can be shown in figure 2 .

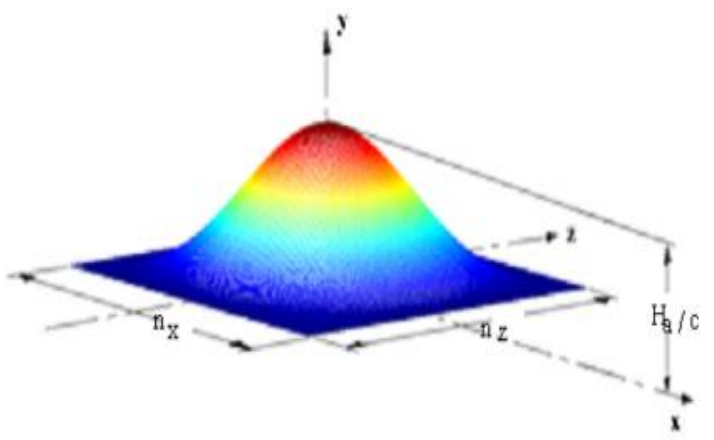

Fig. 2. Roughness profile

\section{Boundary Conditions}

Reynold's boundary condition has been adopted in the present work as follows:

$$
\bar{P}=0 \text { and } \frac{\partial \bar{P}}{\partial \theta}=0 \text { at } \theta=\theta_{c}
$$

In the cavitation zone the negative pressure was assumed to be zero. The pressure at both ends of the bearing equal to the atmospheric pressure, i.e: $\bar{P}(\theta, 0)=\bar{P}(\theta, 1)=0$

The oil feeding pressure can be taken as atmospheric pressure.

\subsection{Bearing characteristics}

The total load capacity of the journal bearing is defined as [4]:

$$
W=\sqrt{W_{r}^{2}+W_{t}^{2}}
$$

where $W_{r}$ and $W_{t}$ are the load components along and perpendicular to the line of centres respectively

$$
W_{r}=\frac{\bar{W}_{r} \mu L U R^{2}}{C^{2}} \int_{0}^{1} \int_{0}^{2 \pi} \bar{P} \cos \theta d \theta d \bar{z} ;
$$

and

$$
W_{t}=\frac{\bar{W}_{t} \mu L U R^{2}}{C^{2}} \int_{0}^{1} \int_{0}^{2 \pi} \bar{P} \sin \theta d \theta d \bar{z} .
$$

where $\mathrm{r}$ and $\mathrm{t}$ denoted along and perpendicular to the line of centres respectively.

The bearing side-leakage flow can be obtained from the following equation: 
$Q_{s}=\left.\frac{U R^{2} C}{L} \int_{0}^{2 \pi} \frac{\bar{J}(\bar{h}, \bar{l})}{12} \frac{\partial \bar{P}}{\partial \bar{z}}\right|_{\bar{z}=0} d \theta$

The viscous friction force of a shearing film fluids can be expressed as [4]:

$$
F_{f}=\frac{\mu \mathrm{LUR}}{C} \int_{0}^{1} \int_{0}^{2 \pi}\left(\frac{1}{\bar{h}}+\frac{\bar{h}}{2} \frac{\partial \bar{P}}{\partial \theta}\right) d \theta d \bar{z}
$$

The coefficient of the friction can be calculated by using the relation:

$$
C_{f}=\frac{F_{f}}{W}(R / C) \text {. }
$$

\subsection{Solution Procedure}

The problem of the present work was solved by using finite difference method. The governing equations for an elliptical journal bearing with different ellipticity factor lubricated with couple stress fluid with different couple stress parameters were solved by using a suitable prepared FORTRAN computer program. The Reynolds' equation was discretized using central difference technique as follows:

$$
\begin{aligned}
& \frac{\partial \bar{I}(\bar{h}, \bar{l})}{\partial \theta} \frac{\partial \bar{P}}{\partial \theta}+\bar{I}(\bar{h}, \bar{l}) \frac{\partial^{2} \bar{P}}{\partial \theta^{2}}+(R / L)^{2} \frac{\partial \bar{I}(\bar{h}, \bar{l})}{\partial \bar{z}} \frac{\partial \bar{P}}{\partial \bar{z}}+ \\
&(R / L)^{2} \bar{I}(\bar{h}, \bar{l}) \frac{\partial^{2} \bar{P}}{\partial \bar{z}^{2}}=6 \bar{\mu} \frac{\partial \bar{h}}{\partial \theta} \\
& \frac{\partial \bar{h}}{\partial \theta}=\frac{\bar{h}_{(i+1, k)}-\bar{h}_{(i-1, k)}}{2 \Delta \theta} \\
& \frac{\partial \bar{I}}{\partial \theta}=\frac{\bar{I}_{(i+1, k)}-\bar{I}_{(i-1, k)}}{2 \Delta \theta} \\
& \frac{\partial \bar{I}}{\partial \bar{z}}=\frac{\bar{I}_{(i, k+1)}-\bar{I}_{(i, k-1)}}{2 \Delta \bar{z}} \\
& \frac{\partial \bar{P}}{\partial \theta}=\frac{\bar{P}_{(i+1, k)}-\bar{P}_{(i-1, k)}}{2 \Delta \theta} \\
& \frac{\partial \bar{P}}{\partial \bar{z}}=\frac{\bar{P}_{(i, k+1)}-\bar{P}_{(i, k-1)}}{2 \Delta \bar{z}} \\
& \frac{\partial^{2} \bar{P}}{\partial \theta^{2}}=\frac{\bar{P}_{(i+1, k)}-2 \bar{P}_{(i, k)}+\bar{P}_{(i-1, k)}}{\Delta \theta^{2}} \\
& \frac{\partial^{2} \bar{P}}{\partial \bar{z}^{2}}=\frac{\bar{P}_{(i, k+1)}-2 \bar{P}_{(i, k)}+\bar{P}_{(i, k-1)}}{\Delta \bar{z}^{2}}
\end{aligned}
$$

Substitute the above discrete forms of the Reynolds equation terms in the equation (15) to get:

$$
\bar{P}_{(i, k)}=\frac{\left\{\begin{array}{l}
\left(A_{1}+A_{2}\right) \bar{P}_{(i+1, k)}+\left(A_{2}-A_{1}\right) \bar{P}_{(i-1, k)}+ \\
\left(A_{3}+A_{4}\right) \bar{P}_{(i, k+1)}+\left(A_{4}-A_{3}\right) \bar{P}_{(i, k-1)}-A_{5}
\end{array}\right\}}{2\left(A_{2}+A_{4}\right)}
$$

where:

$$
\begin{aligned}
& A_{1}=\frac{\bar{I}_{(i, k+1)}-\bar{I}_{(i, k-1)}}{4 \Delta \theta^{2}} \\
& A_{2}=\frac{\bar{I}_{(i, k)}}{\Delta \theta^{2}} \\
& A_{3}=\left(\frac{R}{L}\right)^{2} \frac{\bar{I}_{(i, k+1)}-\bar{I}_{(i, k-1)}}{4 \Delta \bar{z}^{2}} \\
& A_{4}=\frac{\left(\frac{R}{L}\right)^{2} \bar{I}_{(i, k)}}{\Delta \bar{z}^{2}} \\
& A_{5}=6 \bar{\mu} \frac{\bar{h}_{(i+1, k)}-\bar{h}_{(i-1, k)}}{2 \Delta \theta}
\end{aligned}
$$

The discretized Reynolds equation was solved together with the modified film thickness equation to include the effect of surface protrusion using direct iterative procedure with successive over relaxation factor. The solution was obtained for the previously mentioned boundary conditions. It was assumed that the cavitation effect is neglected and the pressure at the cavitation zone equal to zero. The iterations for the pressure loop are stopped when the following criterion for the pressure is obtained:

$$
\frac{\left(\sum P_{i, j}\right)_{n-1}-\left(\sum P_{i, j}\right)_{n}}{\left(\sum P_{i, j}\right)_{n}} \leq 0.0001
$$

where $\mathrm{n}$ and $\mathrm{n}-1$ are the next and previous iterations respectively. As the oil film pressure is obtained it can be integrated to calculate the load carried by the bearing and the different bearing parameters.

\section{RESULTS AND DISCUSSION}

Static characteristics for elliptic journal bearing with various ellipticity factors of (1 and 1.5) lubricated with couple stress lubricant was evaluated in terms of pressure generated in the oil film, load carried by the bearing and its coefficient of friction. Three different values of couple stress fluid factors $\bar{l}=0,0.2$ and 0.4 , eccentricity ratios ranging between 0.2 to 0.7 and micro protrusion height of 0 and 0.3. A validation for the mathematical model was implemented by comparing the predicted oil film pressure distribution obtained in the present work with that obtained by Hussain et al. [24] (1996) for finite length elliptic journal bearing as can be seen in figure (3). A good agreement between the results has been obtained. Figure 4 shows the distribution of pressure for the elliptical journal bearing with different protrusion heights lubricated with couple stress lubricant that has different couple stress parameters working at eccentricity ratio of 0.6 . This figure clearly shows that the oil film pressure generated in two lower and upper lobes and it becomes higher when the bearing lubricated with oil that has higher couple stress parameter. For 
smooth bearing (i.e. $H_{a}=0$ ) it can be noticed that lower lobe maximum oil film pressure increases from 1.8MPa when the bearing lubricated with Newtonian lubricant to $2.2 \mathrm{MPa}$ and $4 \mathrm{MPa}$ for the couple stress lubricated bearing that has parameters of 0.2 and 0.4 respectively. This can be explained by the higher oil viscosity of the bearing in this case. It can also be seen that the effect of surface protrusion is to enhance the oil film pressure. The oil film pressure increases by $17 \%$ for the bearing with surface protrusion height of 0.3 lubricated with Newtonian oil in comparison with that of smooth surface while it becomes $21 \%$ for a bearing lubricated with lubricant that has $\bar{l}=0.2$ and surface protrusion height of 0.3 . This can be assigned to the smaller thickness of the bearing oil film in this case. The effect of bearing ellipticity factor on the pressure distribution of protruded elliptical journal bearing working at an eccentricity ratio of 0.6 can be shown in figure 4 . This figure obviously shows that the oil film pressure decreases for the bearing with higher ellipticity factor. The maximum percentage decrease in maximum pressure at the upper lobe was calculated and found to be $9 \%$ when the ellipticity factor increases from 1 to 1.5. This behavior can be explained by referring to figure 5. This figure clearly shows that the thickness of the oil film increases for the bearing with higher ellipticity factor. This figure also depicts that the non-circularity of the bearing causes in generating two minimum film thicknesses on both sides. The trained of this result is supported by that obtained by Mishra [13]. Three dimensional oil film pressure on the bearing surface for a bearing lubricated with different oils can be shown in figures 6 and 7. These figures clearly depict that two lobes of oil film pressure are produced in elliptical journal bearing with a lower oil film pressure for the bearing lubricated with Newtonian oil in comparison with that lubricated by couple stress lubricant with parameter of 0.2. This assigned to the fact of higher oil viscosity of the couple stress lubricant in comparison with the Newtonian oil.

Figure 8 shows the effect of lubricating smooth and protruded elliptic journal bearing with couple stress fluid that has different couple stress parameters on the load carrying capacity of such bearing. It can be seen that the load carried by the bearing increases when lubricated with couple stress lubricant that has higher parameter due to the higher viscosity of the lubricant. It can also be observed that the effect of surface protrusion is to increase the load carried by bearing. The effect of surface protrusion height becomes mor significant when the bearing working at higher eccentricity ratio since the oil film thickness becomes smaller in this case in comparison with that of smooth surfaces. Considering the combined effect of lubricating the bearing with couple stress fluid and the bearing roughness is clearly noticed when it lubricated with couple stress lubricant that has higher parameter. The effect of protrusion height becomes clear even when the bearing works at lower eccentricity ratios. For example, the load carried by the bearing with protrusion height of 0.3 increases by $18.2 \%$ in comparison with that of

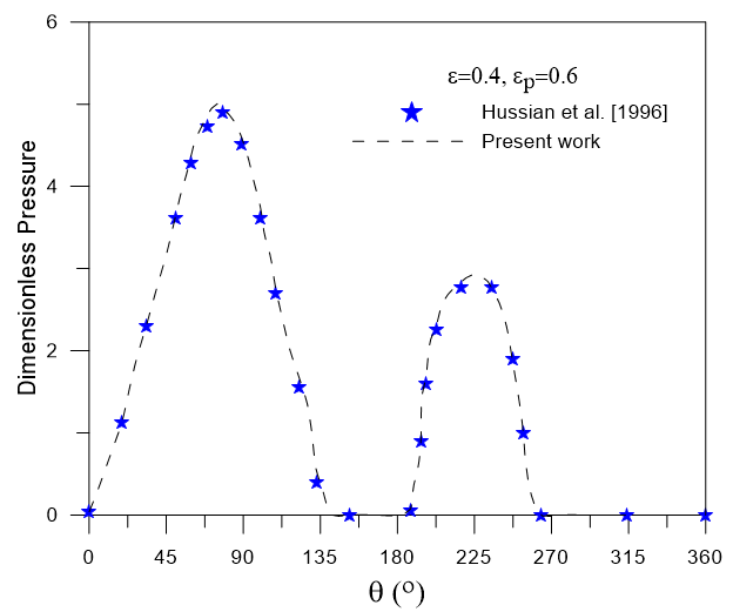

Fig. 3. Comparsion of the oil film pressure with that obtained by Hussain et al. [1996]

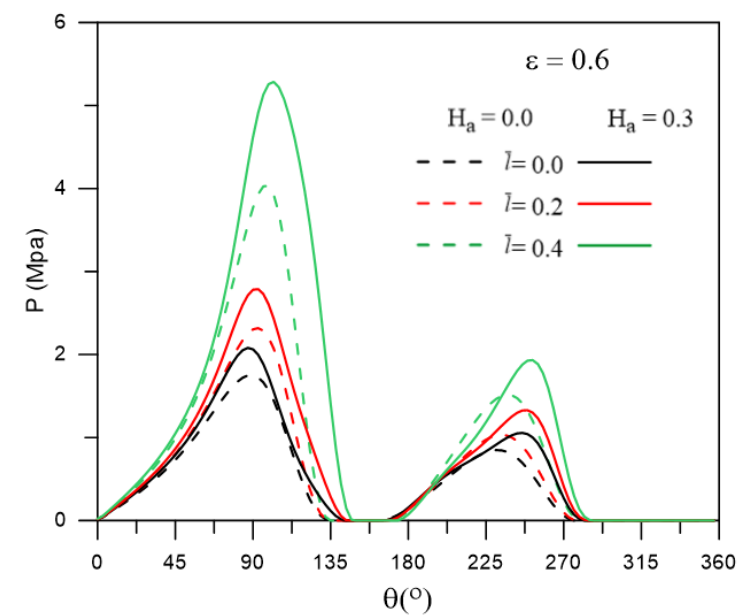

Fig. 4. Effect of couple stress parameter on the pressure distribution of rough and smooth elliptical bearings

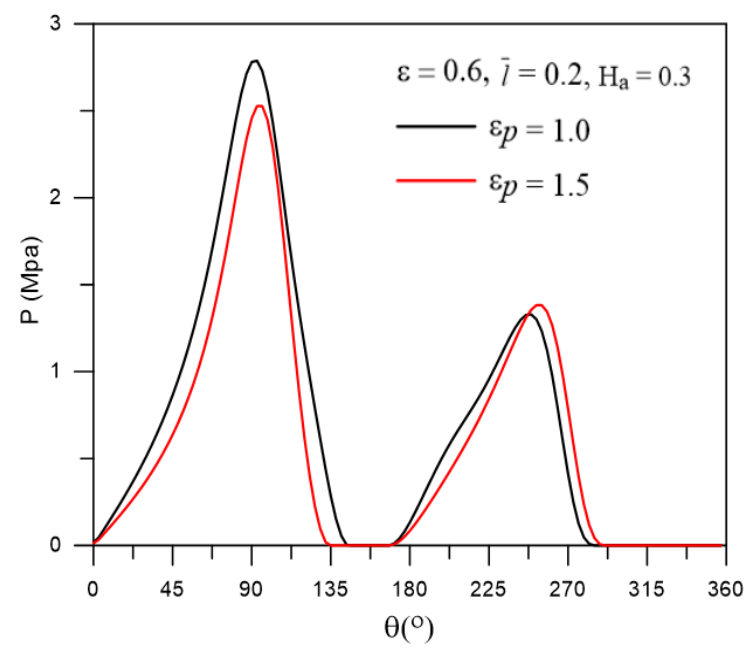

Fig. 5. Effect of ellipticity parameter on the pressure distribution of rough elliptical bearings. 
smooth surfaces lubricated with couple stress lubricant that has couple stress factor of 0.4 and working at eccentricity ratio of 0.5 . The percentage increase becomes higher as the bearing works at higher eccentricity ratios.

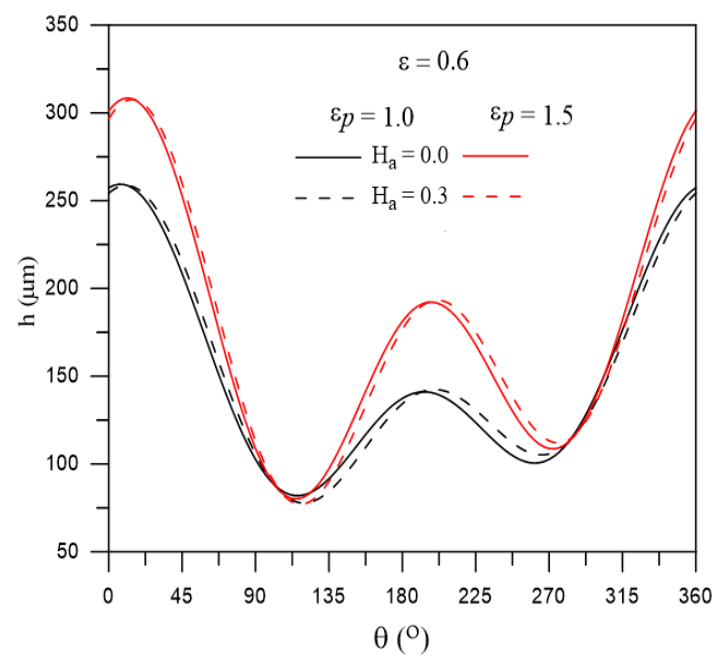

Fig. 6. Effect of ellipticity parameter on the oil film thickness distribution of smooth and rough elliptical bearing.

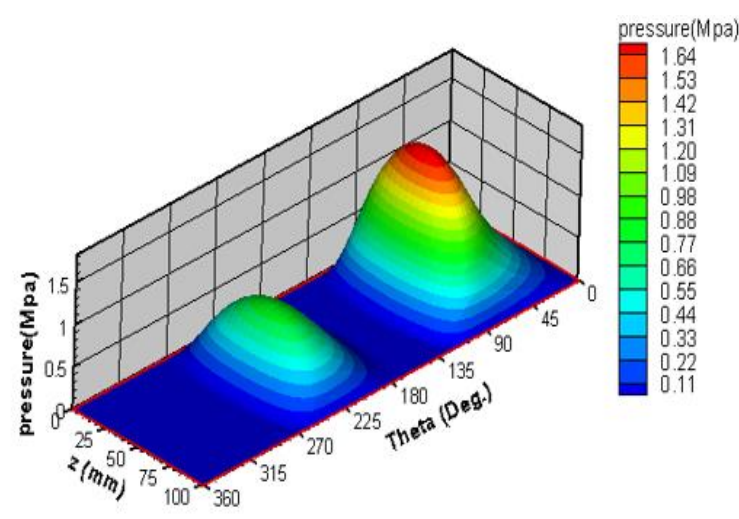

Fig. 7. Three-dimensional pressure distribution for elliptical journal bearing with ellipticity factor of

$$
1, \varepsilon=0.6, \bar{l}=0, H_{a}=0 \text {. }
$$

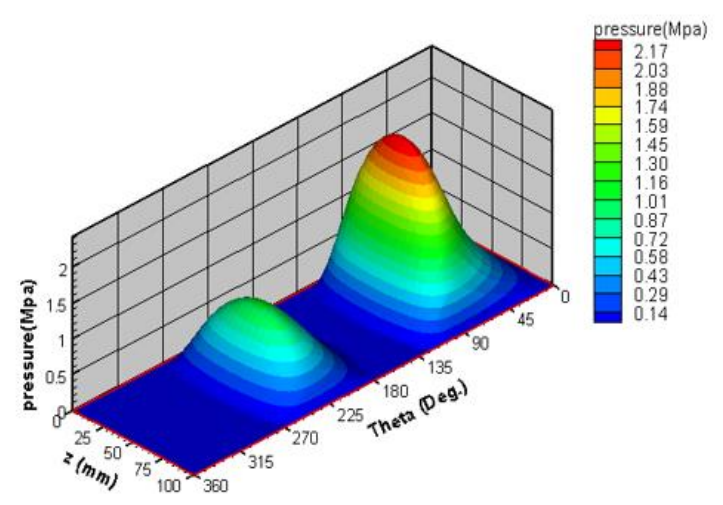

Fig. 8. Three- dimensional pressure distribution for elliptical journal bearing with ellipticity factor of

$$
1, \varepsilon=0.6, \bar{l}=0.2, H_{a}=0 \text {. }
$$

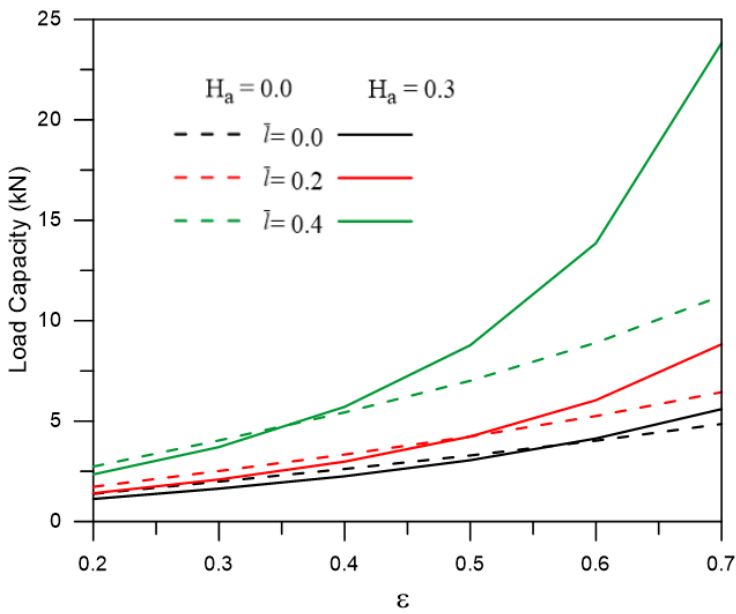

Fig. 9. Effect of couple stress parameter on the load carrying capacity of rough and smooth elliptical bearing.

Figure 9 shows the effect of lubricating the protruded elliptical bearing with couple stress fluid of different CSF parameters. It is observed that the bearing coefficient of friction decreases when the it works at higher eccentricity ratios. The friction coefficient increases when the couple stress fluid with higher parameter used to lubricating the bearing in comparison with that lubricated with Newtonian oil. This can be attributed to the higher viscosity of the oil and hence the friction force induced on the bearing surfaces. This figure also depicts that the protruded bearing with higher surface protrusion has in general higher coefficient of friction in comparison with the bearing of smooth surfaces accept when the bearing works at higher eccentricity ratio when it becomes lower than that of smooth surfaces. This can be explained by the fact of the higher load carried by the bearing in this case. The load carried by the bearing seems to be lower for elliptical bearing with higher ellipticity factor as can be shown in figure 10. This assigned to the larger oil film thickness for the bearing with higher ellipticity factor and higher sensitivity of the pressure generated to the invers of the third power of the oil film thickness. This figure also shows that the effect of ellipticity factor becomes more considerable when the bearing works at higher eccentricity ratio since the percentage decrease in load carrying capacity increases from $23 \%$ to $43 \%$ for the bearing works at eccentricity ratios of 0.4 and 0.7 respectively.

The coefficient of friction seems to be lower for the bearing with lower ellipticity factor as can be shown in figure 11. This can be related to the increase of the load carried by the bearing with ellipticity factor of (1) in comparison with the bearing with ellipticity factor of (1.5). For example, the percentage decrease in the coefficient of friction reaches $30 \%$ for the elliptical bearing with ellipticity factor of 1 in comparison with that of ellipticity factor of 1.5 when it works at eccentricity ratio of 0.5 . 


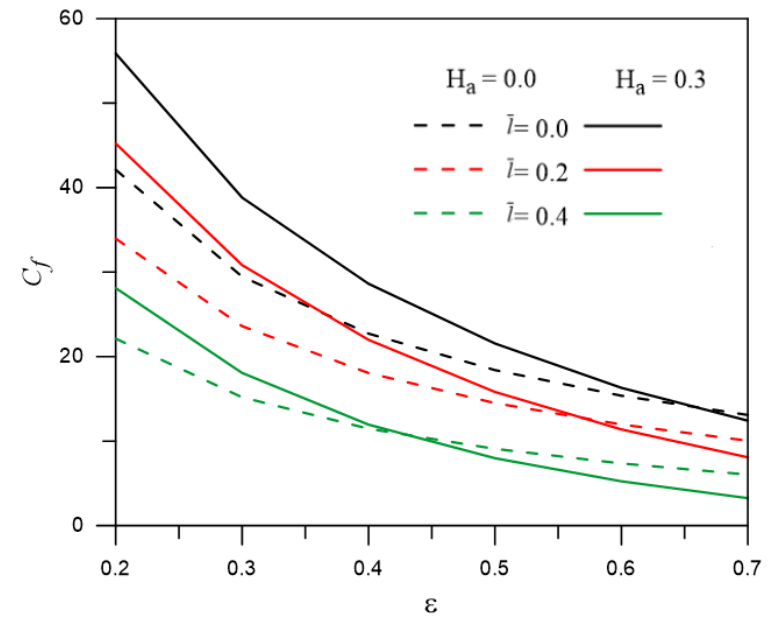

Fig. 10. Effect of couple stress parameter on the coefficient of friction of rough and smooth elliptical bearing

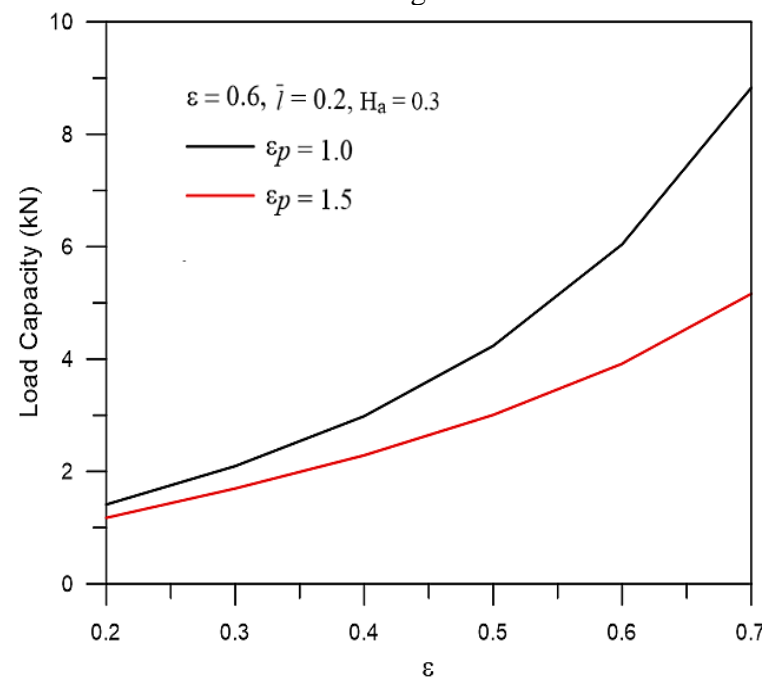

Fig. 11. Effect of ellipticity parameter on the load carrying capacity of rough elliptical bearing.

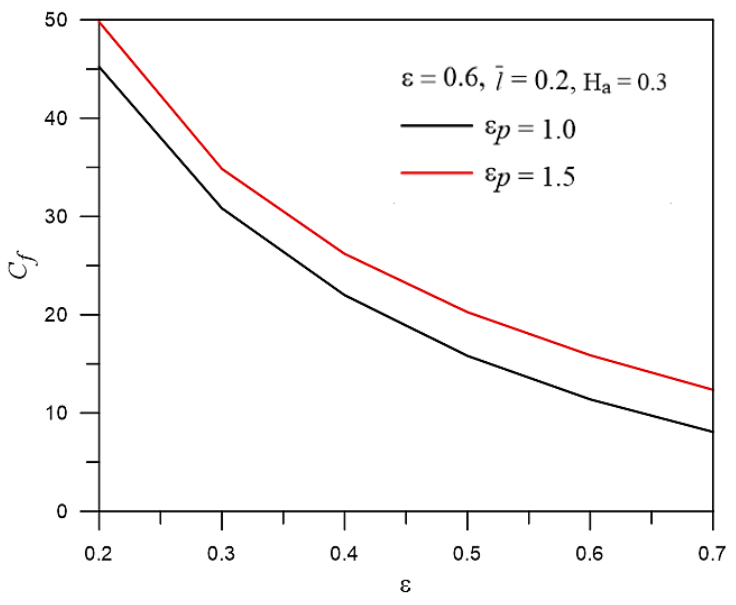

Fig. 12. Effect of ellipticity parameter on the friction coefficient of rough elliptical bearing

\section{CONCLUSIONS}

Theoretical investigation to the static characteristics of protruded elliptical bearing lubricated with lubricant containing long chain additives has been carried out using suitable mathematical model. The effect of protrusion height, the bearing ellipticity factor and the couple stress lubricant parameter on the static bearing performance parameters have been studied and presented. The following are the most important conclusions:

1. Two lobes of positive pressure was found for the elliptical bearing which makes the cavitation zoee (non-effective zone) is smaller in comparison with the conventional journal bearing.

2. The pressure and the load carrying capacity increase for the bearing with higher protrusion height. The maximum oil film pressure for the bearing with protrusion height $\mathrm{H}_{\mathrm{a}}=0.3$ increases by $17 \%$ in comparison with that of smooth surface $\mathrm{Ha}=0$ when lubricated by pure oil while it becomes $21 \%$ and $30 \%$ for couple stress lubricated bearing with couple stress parameters of 0.2 and 0.4 respectively.

3. The combined effect of higher ellipticity factor and protrusion height lead to increase the coefficient of friction. For example, the coefficient of friction increases by $30 \%$ when the bearing with ellipticity factor of (1.5) works at an eccentricity ratio of 0.5 in comparison with that. of ellipticity factor of (1).

4. The load carried by the bearing decreases by $30 \%$ to $43 \%$ for the bearing works at eccentricity ratios of 0.4 and 0.7 respectively when ellipticity factor is (1.5) in comparison with that has ellipticity factor of (1).

5. Protruded elliptical journal bearing shows higher friction coefficient in comparison with the smooth one for a range of working eccentricity ratios. This range was found to be 0.4,0.6 and 0.7 for the bearing lubricated with Newtonian and couple stress lubricant that has parameters of 0.2 and 0.4 respectively.

\section{REFERENCES}

1. Xiao-Li Wang, Ke-Qin Zhu, Shi-Zhu Wen. Thermohydrodynamic analysis of journal bearings lubricated with couple stress fluids. Tribology International. 2001; 34: 335-343. https://doi.org/10.1016/S0301679X(01)00022-6

2. Yan-Yan Ma, Wei-Hua Wang, Xian-Hua Cheng. A study of dynamically loaded journal bearings lubricated with non-Newtonian couple stress fluids. Tribology Letters. 2004;17(1):69-74. https://doi.org/10.1023/B:TRIL.0000017420.44627.63

3. Hsiu-Lu Chiang, Cheng-Hsing Hsu, Jaw-Ren Lin. Lubrication performance of finite journal bearings considering effects of couple stresses and surface roughness. Tribology International. 2004; 37: 297 307. https://doi.org/10.1016/j.triboint.2003.10.005

4. Crosby WA, Chetti B. The static and dynamic characteristics of a two-lobe journal bearing lubricated with couple-stress fluid. Tribology Transactions. 2009;52:262-268. https://doi.org/10.1080/10402000802527773 
5. Faisal Rahmani, Jayanta K. Dutt, Raj K. Pandey. performance behavior of elliptical-bore journal bearings lubricated with solid granular particulates. Particuology. 2016;27:51-60. https://doi.org/10.1016/j.partic.2015.12.009

6. Chetti B. Elastohydrodynamic Analysis of an offset journal bearing lubricated with couple stress fluid. International Journal of Engineering Research in Africa. 2010;2:53-62.

https://doi.org/10.4028/www.scientific.net/JERA.2.53

7. Amit Chauhan, Rakesh Sehgal, Rajesh Kumar Sharma. Thermo hydrodynamic analysis of elliptical journal bearing with different grade oils. Tribology International. 2010;43:1970-1977. https://doi.org/10.1016/j.triboint.2010.03.017

8. Amit Singla, Amit Chauhan. Experimental evaluation of lubricating oil film pressure and temperature of elliptical non-circular journal bearing profile. Proceedings of 2015; RAECS UIET Panjab University Chandigarh. https://doi.org/10.1109/RAECS.2015.7453334

9. Singla A, Chauhan A. Evaluation of oil film pressure and temperature of an Elliptical Journal Bearing - An Experimental Study. Tribology in Industry. 2016;38(1):74-82.

https://www.researchgate.net/deref/https $\% 3 \mathrm{~A} \% 2 \mathrm{~F} \% 2$ Fcreativecommons.org\%2Flicenses $\% 2 \mathrm{Fby}-$ $\mathrm{nc} \% 2 \mathrm{~F} 4.0 \% 2 \mathrm{~F}$

10. Amit Chauhan, Rakesh Sehgal, Rajesh Kumar Sharma. Investigations on the thermal effects in noncircular journal bearings. Tribology International. 2011;44:1765-1773.

https://doi.org/10.1016/j.triboint.2011.06.028

11. Amit Singla, Amit Chauhan. Experimental study for performance evaluation of steadily loaded true elliptical and orthogonally displaced non-circular journal bearing profiles. Industrial Lubrication and Tribology. 2016;68(6):702-711. https://doi.org/10.1108/ILT-10-2015-0155

12. Rajeev Kumar Dang, Amit Chauhan, Dhami SS. Static thermal performance evaluation of elliptical journal bearings with nano-lubricants. Proc IMechE Part J: Journal of Engineering Tribology 2020;2:113. https://doi.org/10.1177\%2F1350650120970742

13. Prakash Chandra Mishra. Isothermal analysis of elliptic bore journal bearing considering isotropic roughness. International Journal of Mechanical \& Mechatronics Engineering. 2012; 12(4): 24-31.

14. Mishra PC. Analysis of a rough elliptic bore journal bearing using expectancy model of roughness characterization. Tribology in Industry. 2014; 36(2): 211-219. http://www.tribology.fink.rs/

15. EL-Said AKH, EL-Souhily BM, Crosby WA, ELGamal HA. The performance and stability of threelobe journal bearing textured with micro protrusions. Alexandria Engineering Journal. 2017; 56(4):423432. https://doi.org/10.1016/j.aej.2017.08.003

16. Marco TC. Faria. Finite element analysis of oillubricated elliptical journal bearings. International Journal of Mechanical, Aerospace, Industrial and Mechatronics Engineering. 2015; 9(5): 628-633

17. Boualem Chetti. The effect of turbulence on the performance of a two-lobe journal bearing lubricated with a couple stress fluid. Industrial Lubrication and Tribology. 2016;68(3):336-340. https://doi.org/10.1108/ILT-06-2015-0072

18. Maulik Ukania, Puneet Mathur. The static and dynamic characteristic of elliptical journal bearing lubricated with couple-stress fluid. International
Conference on Advancements in Computing \& Management (ICACM-2019).

https://dx.doi.org/10.2139/ssrn.3462231

19. Vishwanath B. Awati, Ashwini Kengangutti. Surface roughness effect on thermohydrodynamic analysis of journal bearings lubricated with couple stress fluids. Nonlinear Engineering. 2019;8:397-406. https://doi.org/10.1515/nleng-2018-0017

20. Punit Kumar, Rohilla, Rajive Verma, Suresh Verma. Performance analysis of couple stress fluid operated elastic hydrodynamic journal bearing, Tribology on line. 2019;13(3): 143-154. https://doi.org/10.2474/trol.14.143

21. Tyrone Dass, Sreedhara Rao Gunakala, Donna M.G. Comissiong. The combined effect of couple stresses, variable viscosity and velocity-slip on the lubrication of finite journal bearings. Ain Shams Engineering Journal. 2020;11:501-518. https://doi.org/10.1016/j.asej.2020.01.002

22. Navin Kumar, Akash Shukla, Sanjay Bansal, Chandra B Khatri, Gannath D Thakre, Saurabh K Yadav, Satish C Sharma, Suraj P Harsha. Thermohydrodynamic simulation study of twin-groove elliptical (two-lobe) journal bearing of steam turbine with experimental investigations. Proc IMechE Part J: Journal Engineering Tribology. 2020:1-20. http://dx.doi.org/10.1177/1350650120973798

23. Bhaskera B, Seetharamaiahb N, Ramesh Babu P. Experimental investigations on elliptical journal bearing using hydrol 68 lubricating oil. AIP Conference Proceedings. 2021;2317:030023. https://doi.org/10.1063/5.0036458

24. Hussain A, Mistry K, Biswas S. Thermal Analysis of Noncircular Bearings. Transactions of the ASME. 1996;18:246-254. https://doi.org/10.1115/1.2837086

Received 2021-02-10

Accepted 2021-06-05

Available online 2021-06-10

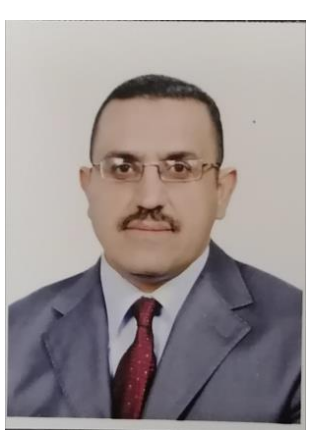

Basim A. Abass was born in Baghdad, Iraq in 1962. He received the B.S. degrees in Mechanical engineering from the University of Baghdad, in 1984, M.Sc. in mechanical engineering in 1989 from the University of Baghdad and the Ph.D. degree in mechanical engineering in 1999.

$\mathrm{He}$ was an assitant proffesor from 2009 till now at the university of Babylon, college of engineering, Mechanical engineering department.

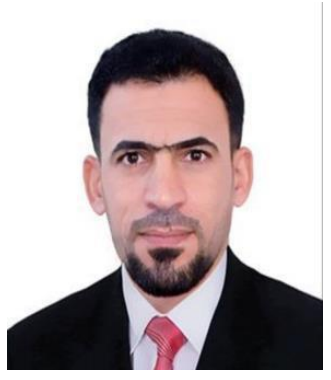

Mushrek A. Mahdi was born in Baghdad, Iraq in 1989. He received the B.S. degrees in Mecha-nical engineering from the University of Babylon, in 2012, M.Sc. mechanical engineering in 2015 from the University of Babylon $\mathrm{He}$ was an assitant lecturer from 2016 till now at the University of Babylon, college of engineering/Almusayab, Automobile engineering department. 